Pacific Journal of Mathematics

A CLASS OF MAXIMAL TOPOLOGIES 


\title{
A CLASS OF MAXIMAL TOPOLOGIES
}

\author{
Douglas E. Cameron
}

In this note, we characterize maximal topologies of a class of topological properties which include lightly compact spaces and $Q H C$-spaces and, when restricted to completely regular spaces, pseudocompact spaces. In addition we prove some results relating maximal lightly compact and maximal pseudocompact spaces.

A. B. Raha [12] has shown that maximal lightly compact spaces are submaximal as are maximal pseudocompact spaces, and Douglas E. Cameron [6] has characterized maximal $Q H C$-spaces and shown these to be submaximal. In Tychonoff spaces, lightly compact and pseudocompact are equivalent; and in Hausdorff spaces, $Q H C$ and $H$-closed are equivalent. We shall show that the maximal topologies of a class of topologies which include lightly compact and $Q H C$ are submaximal and $T_{1}$ spaces.

The topological space with topology $\tau$ on set $X$ shall be denoted by $(X, \tau)$, the closure of a subset $A$ of $X$ with respect to $\tau$ is $\mathrm{cl}_{\tau} A$ and the interior of $A$ with respect to $\tau$ is int ${ }_{\tau} A$, the complement of $A$ with respect to $X$ is $X-A$, the relative topology of $\tau$ on $A$ is $\tau \mid A$, and the product of spaces $\left(X_{\alpha}, \tau_{\alpha}\right)$ for $\alpha \in \mathfrak{U}$ is $\left(\pi_{\mathfrak{I}} X_{\alpha}, \pi_{\mathfrak{I}} \tau_{\alpha}\right)$.

A topological space $(X, \tau)$ with property $R$ is maximal $R$ if whenever $\tau^{\prime}$ is stronger than $\tau\left(\tau^{\prime} \supset \tau\right)$, then $\left(X, \tau^{\prime}\right)$ does not have property $R$. In [5] it was shown that for a topological property $R,(X, \tau)$ is maximal $R$ if and only if every continuous bijection from a space $(Y, \tau)$ with property $R$ to $(X, \tau)$ is a homeomorphism. A topological space $(X, \tau)$ for which there exists a stronger maximal $R$ topology is said to be strongly $R$. For $A \subseteq X$ the topology $\tau(A)$ with subbase $\tau \cup\{A\}$ is the simple expansion of $\tau$ by $A$.

We shall restrict our study to topological properties which satisfy some or all of the following:

P-1: contractive (preserved by continuous surjections)

P-2: regular closed hereditary

P-3: semi-regular (A topological property $R$ is semi-regular if $(X, \tau)$ has property $R$ if and only if $\left(X, \tau_{S}\right)$ has property $R$ where $\tau_{S}$ is the semi-regularization of $\tau$.)

P-4: contagious (A topological property $R$ is contagious if 
whenever a dense subset of a space has property $R$, the entire space has property $R[8])$.

P-5: finitely unionable (If $(X, \tau)$ is a topological space, $A_{i}=X$, $i=1, \cdots, n$ are subsets which have property $R$, then $\bigcup_{i=1}^{n} A_{i}$ has property $R$ ).

Definition 1. Two topologies $\tau$ and $\tau^{\prime}$ on $X$ are ro-equivalent if $\tau_{S}=\tau^{\prime}{ }_{S}$.

THEOREM 1. An expansion $\tau^{\prime}$ of $\tau$ is ro-equivalent to $\tau$ if and only if $\mathrm{cl}_{\tau^{\prime}} U=\mathrm{cl}_{\tau} U$ for all $U \in \tau^{\prime}[\mathbf{1 0}]$.

THEOREM 2. If a topological property $R$ satisfies $\mathrm{P}-3$, then a maximal $R$ topology is submaximal.

Proof. This follows from the properties of P-3 and the fact that every topological space has a stronger submaximal space with the same semiregularization [3].

COROLlary 1. If a topological property $R$ satisfies $\mathrm{P}-3$, then maximal $R$ topologies are $T_{D}$.

THEOREM 3. If topological property $R$ satisfies $\mathrm{P}-1-\mathrm{P}-5$ a submaximal space $(X, \tau)$ is maximal $R$ if and only if for any $A \subseteq X$, such that both $X$-int $A$ and $A$ have property $R$, then $A$ is closed.

Proof. If $(X, \tau)$ is submaximal and not maximal $R$, then there is $\tau^{\prime} \supset \tau$ such that $\tau_{S}^{\prime} \neq \tau_{S}$ and $\left(X, \tau^{\prime}\right)$ has property $R$. Therefore there is $U \in \tau^{\prime}$ such that $\mathrm{cl}_{\tau} U \supset \mathrm{cl}_{\tau^{\prime}} U$ and thus $\mathrm{cl}_{\tau^{\prime}} U$ is not $\tau$-closed. $\mathrm{cl}_{\tau^{\prime}} U$ and $\mathrm{cl}_{\tau^{\prime}}\left(X-\mathrm{cl}_{\tau^{\prime}} U\right)$ are $\tau^{\prime}$ regular closed and thus are $\tau^{\prime}$ and $\tau$ subspaces with property $R$.

By P-4, $\operatorname{cl}_{\tau}\left(\mathrm{cl}_{\tau^{\prime}}\left(X-\mathrm{cl}_{\tau^{\prime}} U\right)\right)=\mathrm{cl}_{\tau}\left(X-\mathrm{cl}_{\tau^{\prime}} U\right)=X-$ int $_{\tau}\left(\mathrm{cl}_{\tau^{\prime}} U\right)$ has property $R$ with respect to $\tau$.

If $(X, \tau)$ has property $R$ and there is a nonclosed subset $A \subseteq X$ such that both $A$ and $X-$ int $_{\tau} A$ have property $R$, then the topology $\operatorname{cl}_{\tau}(X-$ $A$ ) has property $R$. Since every dense subset of a submaximal space is open, $(X-A) \cup$ int $_{\tau} A$ is $\tau$ open implying $\tau|B=\tau(X-A)| B$ where $\mathrm{cl}_{\tau}(X-A)=B$. Also $\tau|A=\tau(X-A)| A$ so both $A$ and $B$ are $\tau(X-A)$ subspace with property $R$ and by $\mathrm{P}-5,(X, \tau(X-A))$ has property $R$ since $X=A \cup B$, thus $(X, \tau)$ is not maximal $R$.

COROllary 2. A submaximal space satisfying P-1-P-5 with property $R$ in which every subspace with property $R$ is closed is maximal $R$. 
THEOREM 4. If property $R$ satisfies $\mathrm{P}-1-\mathrm{P}-5$ and all one point sets have property $R$, then maximal $R$ spaces are $T_{1}$.

Proof. Let $(X, \tau)$ be submaximal $R$. If for $x_{0} \in X,\left\{x_{0}\right\} \notin \tau$ then $X-\left\{x_{0}\right\}$ is $\tau$-dense therefore is open and so $\left\{x_{0}\right\}$ is closed. If $\left\{x_{0}\right\} \in \tau$ and $\mathrm{cl}_{\tau}\left\{x_{0}\right\}$-int $\operatorname{cl}_{\tau}\left\{x_{0}\right\}=\varnothing$ then since $\left\{x_{0}\right\}$ has property $R, \operatorname{cl}_{\tau}\left\{x_{0}\right\}-\left\{y_{0}\right\}$ has property $R$ for $y_{0} \neq x_{0}$ by P-4. Since $\left\{y_{0}\right\} \notin \tau, \mathrm{cl}_{\tau}\left\{y_{0}\right\}=\left\{y_{0}\right\}$, and the free union of $X-\mathrm{cl}_{\tau}\left\{x_{0}\right\},\left\{y_{0}\right\}$, and $\operatorname{cl}_{\tau}\left\{x_{0}\right\}-\left\{y_{0}\right\}$ has property $R$ and is finer than $(X, \tau)$ which is a contradiction since $(X, \tau)$ is maximal $R$. If $\operatorname{cl}_{\tau}\left\{x_{0}\right\}-$ int $_{\tau} \operatorname{cl}_{\tau}\left\{x_{0}\right\} \neq \varnothing$, choose $y_{0} \in \operatorname{cl}_{\tau}\left\{x_{0}\right\}-$ int $_{\tau} \operatorname{cl}_{\tau}\left\{x_{0}\right\}$. Then $A=$ $\operatorname{cl}_{\tau}\left\{x_{0}\right\}-\left\{y_{0}\right\}$ has property $R$ and is not closed. $X-$ int $_{\tau} A=$ $\mathrm{cl}_{\tau}\left(X-\mathrm{cl}_{\tau} A\right)$ is regular closed and thus has property $R$. By Theorem 3, $A$ is closed, a contradiction as $\left\{x_{0}\right\} \subseteq A \subsetneq \mathrm{cl}_{\tau}\left\{x_{0}\right\}$.

THEOREM 5 If property $R$ is productive and contractive $(\mathrm{P}-1)$ and $\left(\pi_{\mathfrak{A}} X_{\alpha}, \pi_{\mathfrak{r}} \tau_{\alpha}\right)$ is maximal $R$, then $\left(X_{\alpha}, \tau_{\alpha}\right)$ is maximal $R$ for $\alpha \in \mathfrak{A}$.

Proof. $\quad\left(X_{\alpha}, \tau_{\alpha}\right)$ has property $R$ for $\alpha \in \mathfrak{U}$ since $R$ is contractive; if $\left(X_{\beta}, \tau_{\beta}\right)$ is not maximal $R$ for some $\beta \in \mathfrak{A}$, there is $\tau_{\beta}^{\prime} \supset \tau_{\beta}$ such that $\left(X_{\beta}, \tau_{\beta}^{\prime}\right)$ has property $R$. Then for $\tau_{\alpha}^{\prime}=\tau_{\alpha}$ for $\alpha \neq \beta,\left(\pi_{\mathfrak{g}} X_{\alpha}, \pi_{\mathfrak{q}} \tau_{\alpha}^{\prime}\right)$ has property $R$ which is a contradiction.

QHC-spaces (spaces for which every open cover has a finite subcollection whose closures cover the space) have properties P-1-P-5 and have been studied in detail [6]. QHC-spaces which are Hausdorff are called $H$-closed spaces. Lightly compact spaces (spaces for which every countable open cover has a finite subcollection whose closures cover the space) satisfy P-1-P-5 (See [2] for P-2; [12] for P-3; P-1, P-4, and $\mathrm{P}-5$ are proven as for $\mathrm{QHC}$ ). Lightly compact spaces are called feebly compact in [14, 15]. Pseudocompact spaces satisfy P-1, P-3 [12], P-4 [8] and P-5, but not P-2. However P-2 is satisfied for pseudocompactness in the class of completely regular spaces [9] and maximal pseudocompact spaces are $T_{1}$ [7].

Spaces having properties $P_{1}-P_{5}$ are not necessarily strongly $R$ (QHC-[6]; lightly compact-[12]). However $H$-closed spaces are strongly $H$-closed [10] and a first countable Hausdorff space which is lightly compact is strongly lightly compact. This follows from P-3, the fact that every space is coarser than some submaximal space with the same semi-regularization, the fact that in a first countable Hausdorff space, lightly compact subsets are closed (proven similarly to the same result for first countable, $T_{1}$ countably compact spaces [1]) and Corollary 2. In Tychonoff spaces pseudocompactness is closed hereditary [9], thus we have the following result: 
THEOREM 6. A Tychonoff space is maximal pseudocompact if and only if it is maximal lightly compact.

Proof. In completely regular spaces, pseudocompactness is equivalent to lightly compact [2]; since lightly compact spaces are pseudocompact then a lightly compact maximal pseudocompact space is maximal lightly compact. If not maximal pseudocompact there is $\tau^{\prime} \supset \tau$ such that $\left(X, \tau^{\prime}\right)$ is pseudocompact and therefore there is $A \in \tau^{\prime}-\tau$ such that $(X, \tau(A))$ is pseudocompact and is completely regular [13]. Therefore $(X, \tau(A))$ is lightly compact.

\section{REFERENCES}

1. C. E. Aull, A certain class of topological spaces, Prace. Math., 11 (1967), 49-53.

2. R. W. Ragley, E. H. Connell and J. D. McKnight, Jr., On properties characterizing pseudocom pact spaces, Proc. Amer. Math Soc., 9 (1958), 500-506.

3. Bourbaki, General Topology, Addison Wesley, (1966).

4. Douglas E. Cameron, Maximal pseudocompactness, Pro. General Topology Conf., Emory University, (1970), 26-31.

5. —- Maximal and minimal topologies, Trans. Amer. Math. Soc., 160 (1971), 229-248.

6. — Maximal QHC-spaces, Rocky Mountain J. Math., (to appear).

7. — A note on maximal pseudocompactness, J. Australian Math. Soc., submitted.

8. J. Chew, Contagious properties, Math. Mag., (March 1974), 84-87.

9. I. Glicksberg, Stone -Cech compactifications of products, Trans. Amer. Math. Soc., 90 (1959), 369-382.

10. J. Miodurzewski and L. Rudolf, $H$-closed and extremely disconnected Hausdorff spaces, Dissertationes Mathematical, 66 (1969).

11. J. R. Porter, and J. Thomas, On H-closed and minimal Hausdorff spaces, Trans. Amer. Math Soc., 138 (1969), 159-170.

12. A. B. Raha, Maximal topologies, J. Australian Math. Soc., 15 (1973), 279-290.

13. D. F. Reynolds, Expansions of topologies by locally finite collections, Proc. Gen. Top. Conference, Charlotte (1974) Academic Press.

14. C. T. Scarborough and A. H. Stone, Products of nearly compact spaces, Trans. Amer. Math. Soc., 124 (1966), 131-147.

15. R. M. Stephenson, Jr., Pseudocompact spaces, Trans. Amer. Math. Soc., 134 (1968), 437-448.

Received December 8, 1976 and in revised form January 31, 1977.

The University of AKron

AKRON, OH 44325

AND

OHIO UNIVERSITY (visiting)

ATHENS, OH 45701 



\section{Pacific Journal of Mathematics \\ Vol. 70, No. $1 \quad$ September, 1977}

William H. Barker, Noether's theorem for plane domains with hyperelliptic

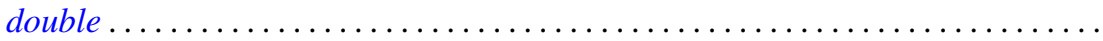

Michael James Beeson, Non-continuous dependence of surfaces of least area on the boundary curve ...................................... 11

Horst Behncke, Functions acting in weighted Orlicz algebras . . . . . . . . . . . . 19

Howard Edwin Bell, A commutativity study for periodic rings . . . . . . . . . . . 29

Peter Botta and Stephen J. Pierce, The preservers of any orthogonal group ....... 37

Douglas S. Bridges, The constructive Radon-Nikodým theorem ............. 51

James Dennis Brom, The theory of almost periodic functions in constructive

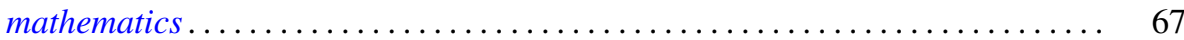

N. Burgoyne and C. Williamson, Semi-simple classes in Chevalley type groups ....

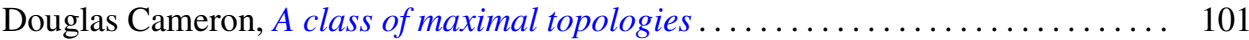

L. Carlitz, Enumeration of doubly up-down permutations . . . . . . . . . . . . . . 105

Paul Robert Chernoff, The quantum n-body problem and a theorem of

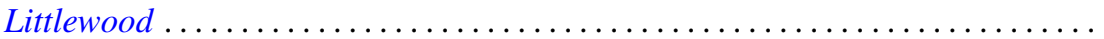

Jo-Ann Deborah Cohen, Locally bounded topologies on $F(X) \ldots \ldots \ldots \ldots \ldots \ldots$

Heinz Otto Cordes and Robert Colman McOwen, Remarks on singular elliptic

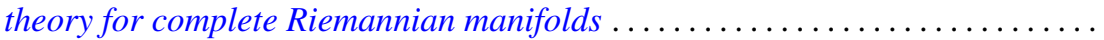

Micheal Neal Dyer, Correction to: "Rational homology and Whitehead

products"

Robert Fernholz, Factorization of Radonifying transformations

Lawrence Arthur Fialkow, A note on quasisimilarity. II ...... . .

Harvey Charles Greenwald, Lipschitz spaces of distributions on the surface of unit

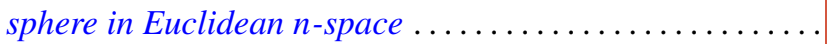

Albrecht Irle, On the measurability of conditional expectations

Tom (Roy Thomas Jr.) Jacob, Matrix transformations involving simple sequence

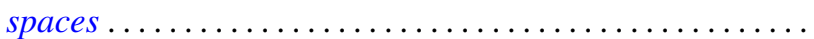

A. Katsaras, Continuous linear maps positive on increasing continuous



Kenneth Kunen and Judith Roitman, Attaining the spread at cardinals of cofinality

Lawrence Louis Larmore and Robert David Rigdon, Enumerating normal bundles

of immersions and embeddings of projective spaces ...... . .

Ch. G. Philos and V. A. Staïkos, Asymptotic properties of nonoscillatory solutions of differential equations with deviating argument .

Peter Michael Rosenthal and Ahmed Ramzy Sourour, On operator algebras containing cyclic Boolean algebras...

Polychronis Strantzalos, Strikt fast gleichgradig-stetige und eigentliche

Aktionen ...

Glenn Francis Webb, Exponential representation of solutions to an abstract

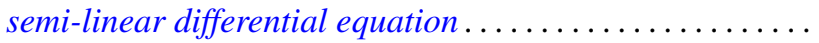

